\title{
Management of a Firmly Lodged Endotracheal Tube: A Case of High Quality Communication
}

\author{
Gabrielle Thompson ${ }^{1}$, Mark Harrison ${ }^{2}$ and Hassan Ahmad ${ }^{2}$ \\ ${ }^{1}$ Department of ENT, Tameside NHS Foundation Trust, UK \\ ${ }^{2}$ Department of Anaesthetics, Tameside NHS Foundation Trust, UK
}

\begin{abstract}
This article describes a case of endotracheal tube firmly lodged in the trachea of a patient due to denuded subglottic cartilage and subglottic swelling. A 30-year female, who had a number of previous intubation episodes, presented with reduced consciousness and was intubated in order to protect and maintain her airway. On day-8 of intubation, it was found that the endotracheal tube was firmly lodged and the patient was taken to theatre for surgical assessment of the airway.

The objective of this clinical case report is to highlight this unusual presentation and outline how it was managed in theatre. Furthermore, this case highlights the importance of a high quality communication using algorithmic surgical management plan to achieve a safe and controlled environment within theatre.
\end{abstract}

Key Words: Teamwork, Communication, Shared airway, Extubation, Endotracheal tube.

How to cite this article: Thompson G, Harrison M, Ahmad H. Management of a Firmly Lodged Endotracheal Tube: A Case of High Quality Communication. J Coll Physicians Surg Pak 2021; 31(05):582-584.

\section{INTRODUCTION}

We report a case of a firmly lodged endotracheal tube (ETT) in the trachea of a patient, who had been intubated for eight days, due to significant subglottic mucosal disease, without any significant subglottic stenosis. Previously reported cases of lodged ETTs have been due to failure of cuff deflation, distorted laryngeal anatomy, manufacturing faults, entanglement with feeding tube and the balloon obstructed by a bite

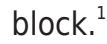

In this case, we highlight the management and the role of high quality communication when dealing with a difficult shared airway. We emphasise the importance of a structured surgical management plan in order to achieve clear communication between the anesthetist, surgeon and theatre team; ${ }^{2}$ as without such inter-professional communication, the team cannot function as one safe unit. ${ }^{3}$ High quality communication between all team members can help identify potential problems earlier, avoiding adverse effects for the patient. ${ }^{2}$ Without adequate communication, we cannot ensure safe and effective clinical practice for our patients.

Correspondence to: Dr. Gabrielle Thompson, Department of ENT, Tameside NHS Foundation Trust, UK

E-mail: gabrielle.thompson1@nhs.net

Received: August 29, 2019; Revised: December 05, 2019;

Accepted: January 31, 2020

DOI: https://doi.org/10.29271/jcpsp.2021.05.582

\section{CASE REPORT}

A 30-year female with insulin-dependent diabetes mellitus presented to Accidentand Emergency (A\&E) Department of this hospital with a Glasgow coma scale (GCS) score of eight. A diagnosis of diabetic ketoacidosis was made. The patient was intubated in order to protect and maintain her airway. The intubation was performed, without difficulty, using an ETT of size 7.0 $\mathrm{mm}$. The patient was shifted to the intensive care unit (ICU) of the hospital.

Past medical history included insulin dependent diabetes mellitus, non-alcoholic fatty liver disease, hypertension and chronic kidney disease, stage 5 , on dialysis (CKD5D). The patient had a number of previous elective intubations.

On day-2 of admission, the ETT was changed, as there was no subglottic suction port. During initial re-intubation attempt, an ETT of size $7.0 \mathrm{~mm}$ could not be easily passed, so an ETT of size $6.0 \mathrm{~mm}$ was placed.

By day-4, it became difficult to pass a suction catheter through the ETT. Laryngoscopy was undertaken, which showed a tight fit of the ETT within the laryngeal inlet, without obvious laryngeal oedema. Flexible bronchoscopy revealed a tight constriction in the lower half of the ETT with a normal appearance of the distal trachea. The intensivist changed the ETT from size $6.0 \mathrm{~mm}$ to $7.0 \mathrm{~mm}$ withoutany difficulty.

In the following days, repeated sedation holds were attempted, but the patient failed the cuff leak tests. On day-8, the ICU team attempted extubation but the ICU nurse was unable to pull the ETT out, despite a completely deflated cuff. The ENT team was engaged and the patient was started on three times daily intra- 
venous dexamethasone $(6.6 \mathrm{mg})$ with the aim to reduce subglottic swelling. ACT scan of the neck was performed, which was reported as normal.

The patient was taken to theatre for assessment of the airway. The ENT surgeon explained the surgical plan to the theatre team to ensure that they were prepared for all potential outcomes, as it was uncertain whether the ETT would be easily removed via the trans-oral route. The plan was drawn as an algorithm on a theatre whiteboard. There was clear description of which surgeon would carry out which role (Figure 1).

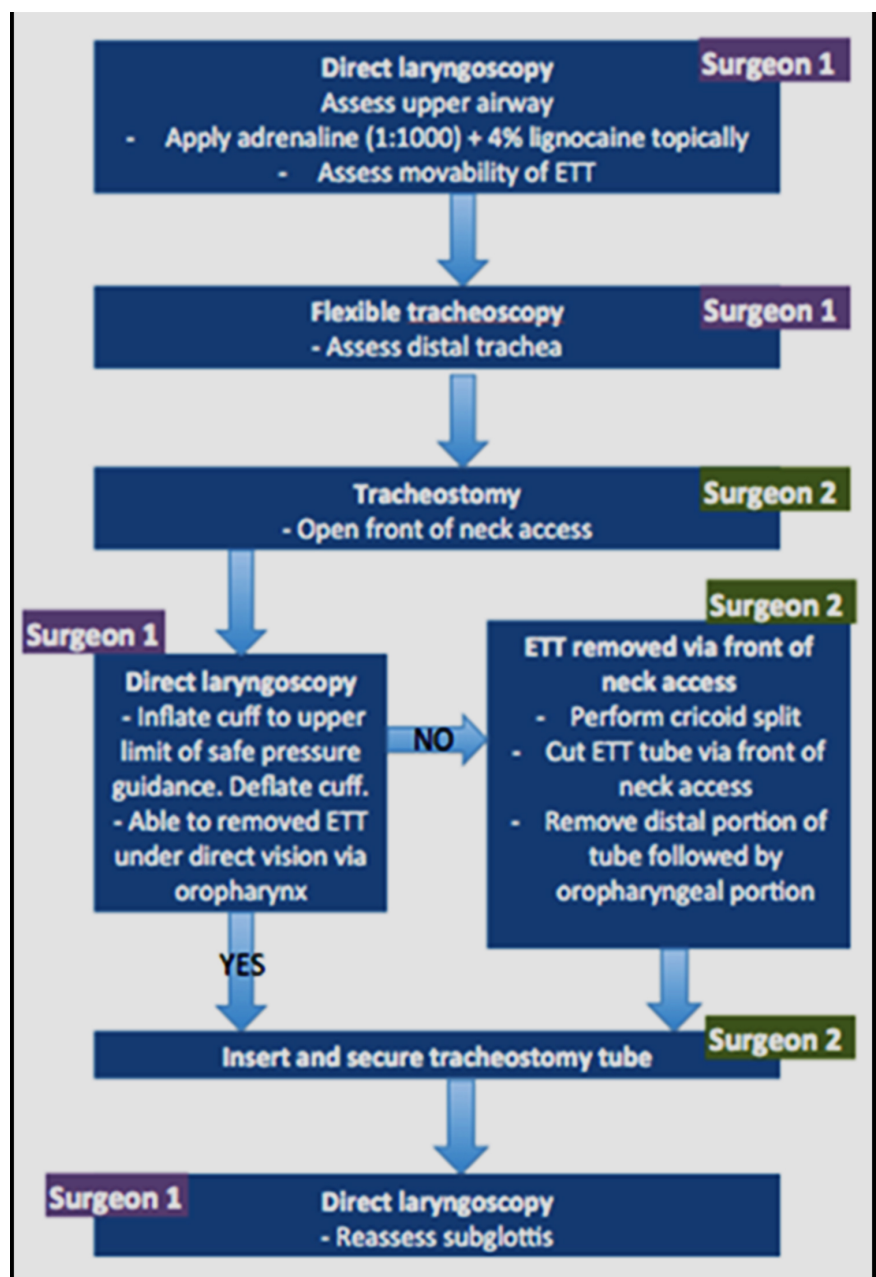

Figure 1: Algorithm of the surgical management plan as drawn on the theatre whiteboard.

On direct laryngoscopy, topical 4\% lidocaine and adrenaline (1:1000) was applied to help reduce any subglottic swelling. Next, a surgical tracheostomy was performed. Once the trachea was exposed, the second surgeon gently inflated and deflated the balloon of the ETT within the subglottis, with pressure guidance. The ETT was removed under direct vision by gentle traction and twisting motions, taking care not to cause damage to the vocal cords. A size $6.0 \mathrm{~mm}$ tracheostomy tube was inserted with no complications. Rigid laryngoscopy was performed which showed denuded cricoid cartilage along with posterior tracheal granuloma formation, without any significant degree of subglottic stenosis (Figure 2).

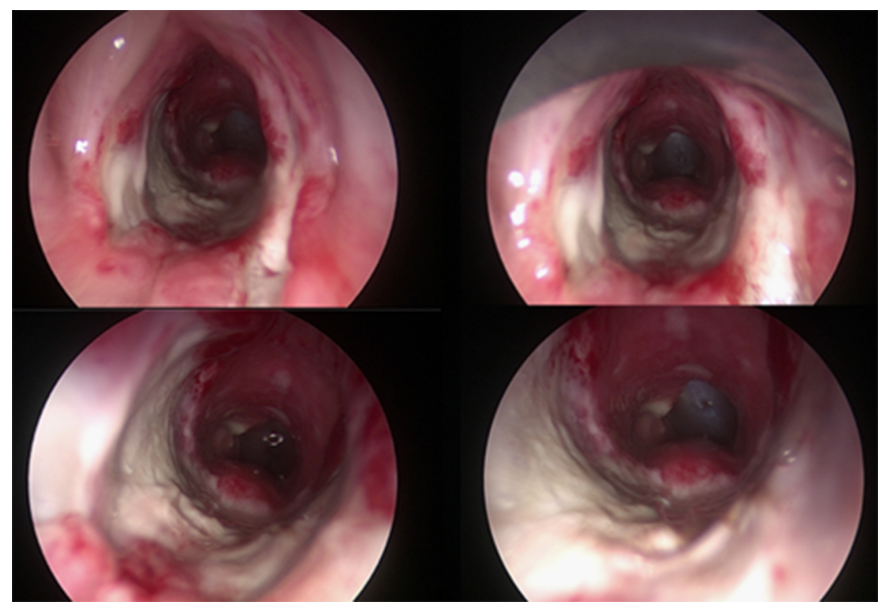

Figure 2: Denuded subglottic cartilage seen on direct laryngo-tracheo-bronchoscopy.

\section{DISCUSSION}

The main point of interest in this case was the inability to remove the ETT from the patients' trachea, despite a fully deflated cuff. The complications of the airway secondary to endotracheal intubation are frequent; though have reduced significantly in the recent years. ${ }^{4}$ Complications can vary in severity depending upon the duration of the endotracheal intubation and the size of the ETT used. Injuries can be due to traumatic intubation or by the use of wide boretubes. ${ }^{4}$

Previous reports of lodged ETTs have been due to failure of cuff deflation, distorted laryngeal anatomy, manufacturing faults, entanglement with feeding tube, and the balloon obstructed by a bite block. ${ }^{1}$ However, in this case, endotracheal intubation was complicated by significant subglottic mucosal disease. There had been two episodes of extubation-reintubation, which may have contributed to the subglottic injury. We feel that size appropriate tubes were used for endotracheal intubation of the patient.

This case also demonstrates the value of high quality communication by the use of an algorithmic management plan in managing such complicated cases. Many studies suggest that patient safety is at least partially reliant upon good quality communication. ${ }^{3}$ Teamwork is essential when caring for a patient with a shared airway. The anaesthetist, surgeon, and theatre team must communicate well with each other and work together to achieve optimal surgical outcomes ${ }^{5}$ without adequate communication, safe and effective clinical practice for patients cannot beensured.

\section{PATIENT'S CONSENT:}

Patient's permission was obtained to publish case report.

\section{CONFLICT OF INTEREST:}

The authors declared no conflict of interest.

\section{AUTHORS' CONTRIBUTION:}

GT, MH, HA: Involved in the case, gathered data, drafted the report and final approval, agreed to be accountable for parts of the work. 


\section{REFERENCES}

1. Panda CK, Karim HM. Too much of anything is bad: An unusual case of a stuck endotracheal tube with deflated cuff. Saudi J Anaesth 2019; 13(2):156-7. doi: 10.4103/sja. SJA_741_18.

2. Jones G. Perioperative teamwork for the patient with a shared airway: A case study. J Perioper Pract 2017; 27(3): 37-42. doi:10.1177/175045891702700301.
3. Weldon S, Korkiakangas T, Bezemer J, Kneebone R. Communication in the operating theatre. Br J Surg 2013; 100(13):1677-88.

4. de Cavalho G, Brito V, Mota L. Laryngeal complications by orotracheal intubation: Literature review. Int Arch Otorhinolaryngol 2012; 16(02):236-45.

5. Patel A. The shared airway. Curr Anaesthe Criti Care 2001; 12(4):213-7. 